\title{
Time and Spacetime: The Crystallizing Block Universe
}

\author{
George F. R. Ellis* and Tony Rothman*† \\ ${ }^{*}$ University of Cape Town, \\ Rondebosch 7701, Cape, South Africa \\ + Princeton University, \\ Princeton, NJ 08544
}

IATEX-ed October 26, 2018

\begin{abstract}
The nature of the future is completely different from the nature of the past. When quantum effects are significant, the future shows all the signs of quantum weirdness, including duality, uncertainty, and entanglement. With the passage of time, after the time-irreversible process of state-vector reduction has taken place, the past emerges, with the previous quantum uncertainty replaced by the classical certainty of definite particle identities and states. The present time is where this transition largely takes place, but the process does not take place uniformly: Evidence from delayed choice and related experiments shows that isolated patches of quantum indeterminacy remain, and that their transition from probability to certainty only takes place later. Thus, when quantum effects are significant, the picture of a classical Evolving Block Universe ('EBU') cedes place to one of a Crystallizing Block Universe ('CBU'), which reflects this quantum transition from indeterminacy to certainty, while nevertheless resembling the EBU on large enough scales.
\end{abstract}

Keywords: Space-time, quantum uncertainty, arrow of time, block universe.

\section{The Nature of Space Time: Emergent universes}

According to the spacetime view associated with both special and general relativity, time, in a real sense, is little more than an illusion. Given data at an arbitrary instant, we assume that everything occurring at a later or earlier time is determined, evolved according to time-reversible local physics. Consequently, nothing is, or can be, special about any particular moment. The standard spacetime diagrams used in relativity enforce such a view: no special status is accorded to the present and indeed the "now" is not usually even denoted on the diagram. Rather, all possible "presents" are simultaneously represented on an equal footing. In a few instances, cosmology takes into account

\footnotetext{
*george.ellis@uct.ac.za

†trothman@princeton.edu
} 
time-irreversible physics (for example nucleosynthesis in the early universe), but the notion of the present as a special time remains absent.

Such a view can be formalized in the idea of a block universe $[1,2,3]$ : space and time are represented as merged into an unchanging spacetime entity, with no particular space sections identified as the present and no evolution of spacetime taking place. The universe just is: a fixed spacetime block. In effect such a representation embodies the idea that time is an illusion: time does not "roll on" in this picture. All past and future times are equally present, and the present "now" is just one of an infinite number. Price [4] and Barbour [5] in particular advocate such a position. ${ }^{1}$ Underlying the idea, as emphasized by Barbour, is that time-reversible Hamiltonian dynamics provides the foundation for physical theory.

By contrast to this view, in a previous paper [6] one of us has argued that the true nature of spacetime is best represented as an Emergent Block Universe (EBU), which adequately represents the differences between the past, present, and future, and which depicts the change from the potentialities of the future to the determinate nature of the past. The main feature of the EBU is an indefinite spacetime, which grows and incorporates ever more events, "concretizing" as time evolves along each world line. That paper, however, was based on a classical view of physics. The present work extends the Emergent Block Universe view to one designed to take account of the quantum nature of physics at microscopic scales. We represent the effects of "quantum weirdness" through a "Crystallizing Block Universe" (CBU), where "the present" is effectively the transition region in which quantum uncertainty changes to classical definiteness. Such a crystallization, however, does not take place simultaneously, as it does in the simple classical picture. Quantum physics appears to allow some degree of influence of the present on the past, as indicated by such effects as Wheeler's delayed choice experiments and Scully's quantum eraser (see the summaries of these effects in $[7,8]$ ). Our CBU picture adequately reflects such effects by distinguishing the transitional events where uncertainty changes to certainty, which may in some cases be delayed till after the apparent "present time."

The CBU is of course a riposte to those proposing an unchanging Block Universe picture as an

\footnotetext{
${ }^{1}$ And see the debates about the idea in the fqxi website for their Nature of Time essay competition, to be found at http://www.fqxi.org/community/forum/category/10; many of the essays there support the Block Universe idea.
} 
adequate representation of spacetime structure on all scales. In our view that picture (based on time-reversible Hamiltonian dynamics) does not represent adequately either the macroscopic arrow of time, nor the unpredictable and time-irreversible quantum measurement process. We first examine the classical case, and then explore how it must be modified by considerations arising from quantum physics.

\section{The Emerging Block Universe: The Classical Picture}

How do we envisage spacetime and the objects in it as time unrolls? To motivate the classical Evolving Block Universe ('EBU') model of reality, consider the following scenario [6]: A massive object has rocket engines attached at each end that allow it to move either left or right. The engines are fired alternately by a computer, which produces firing intervals and burn lengths based on the random decays of a radioactive element. These signals originating from the decaying element thus select the actual spacetime path of the object from the set of all possible paths. Due to the quantum uncertainty in the radioactive decays, the realized path is not determined by initial data at any previous time (see Figure 1).

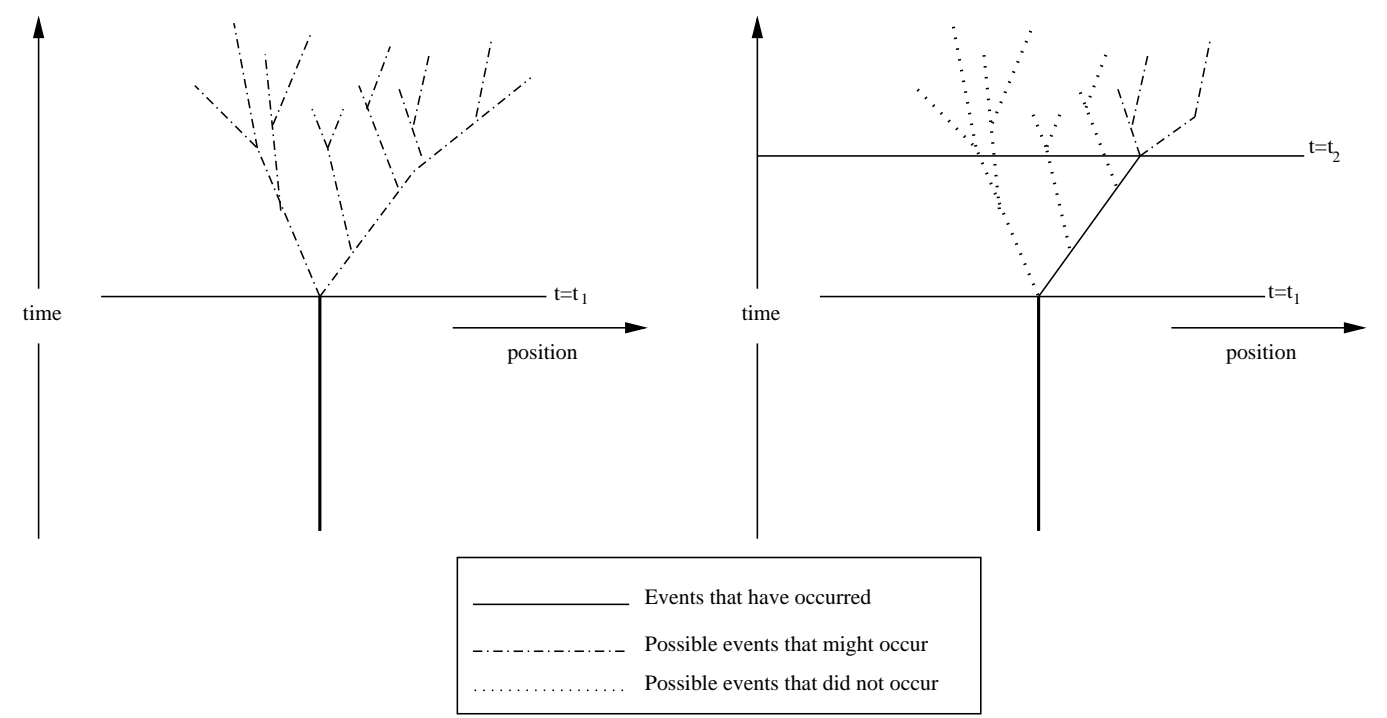

Figure 1: Motion of a particle world line controlled in a random way, so that what happens is determined as it happens. On the left events are determined till time $t_{1}$ but not thereafter; on the right, events are determined till time $t_{2}>t_{1}$, but not thereafter. 
Because the objects are massive and hence produce spacetime curvature, spacetime structure itself is undetermined until the object's motion is determined. Instant by instant, spacetime structure changes from indeterminate to definite. Thus a definite spacetime structure comes into being as time evolves. Spacetime is unknown and unpredictable before it is determined. The Evolving Block Universe model of spacetime represents this situation: time progresses, events take place, and history is shaped. Second by second, one specific evolutionary history out of all possibilities is chosen, takes place, and becomes cast in stone. The basic EBU idea was proposed many years ago by Broad [9], but has not caught on in the physics community. The new element in the more recent presentation [6] is the random element introduced through the irreducible uncertainty of quantum events. This ensures that there is no way the future spacetime can be predicted from the past: what will actually happen is not determined until it happens.

The EBU can be represented through a growing spacetime diagram, in which the passing of time marks the change from indefinite (not yet existing) to definite (having come into being), and in which the present marks the instant at which we can act and change reality (see Figure 2). Even the nature of future spacetime is taken to be uncertain until it is determined at the "now," along with the physical events that occur in it. Unlike special relativity, which operates in matter-free space, the EBU assumes that matter exists and causes spacetime curvature. Depending on the distribution of matter and energy, particular spacetime surfaces and timelike worldlines will be geometrically and physically preferred. The solutions embody broken symmetries of the Einstein field equations: the solutions have less symmetry than the equations of the theory.

One is led to suggest [6] that the transition from present to past does not take place on specific spacelike surfaces; rather it takes place pointwise at each spacetime event, with dynamical processes taking place along timelike world lines (associated with possible particle trajectories), rather than on spacelike surfaces (implicit in usual presentations of the initial value problem in General Relativity).

The proposed view is thus that spacetime is continually extending to the future as events develop along each world line in a way determined by the complex of causal interactions; these shape the future, including the very structure of spacetime itself, in a locally determined (pointwise) way. The 


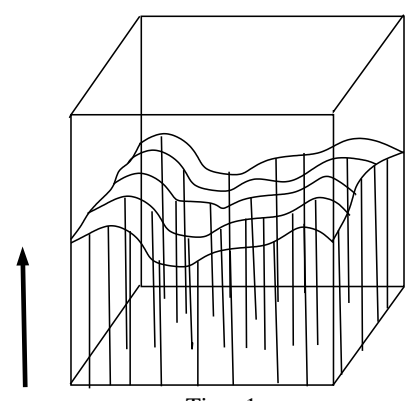

Time 1

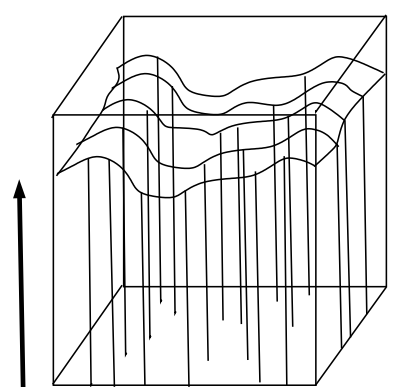

Time 2

Figure 2: An evolving curved space-time picture that takes macro-phenomena seriously. Time evolves along each world line, extending the determinate spacetime as it does so. The particular surfaces have no fundamental meaning and are there for convenience only (one requires some coordinates to describe what is happening). You cannot locally predict uniquely to either the future or the past from data on any 'time' surface (even though the past is already determined). This is true both for physics, and (consequently) for the spacetime itself: the developing nature of spacetime is determined by the evolution (to the future) of the matter in it.

Evolving Block Universe continues evolving along every world line until it reaches its final state, resulting in an unchanging Final Block Universe. One might say that then time has changed into eternity. The future is uncertain and indeterminate until local determinations have taken place at the spacetime event "here and now," designating the present on a world line at a specific instant; thereafter this event is in the past, having become fixed and immutable, with a new event on the world line designating the present. Analysis of the evolution is conveniently based on preferred (matter related) world lines rather than time surfaces. However in order to describe it overall, it will be convenient to choose specific time surfaces for the analysis, but these are a choice of convenience rather than necessity. While the general coordinate invariance invoked in general relativity theory proclaims there are no preferred such surfaces, in any particular solution this is not the case - there will for example usually be preferred spacelike surfaces in any particular cosmological solution.

In summary: The past has taken place and is fixed, and so the nature of its existence is quite different than that of the indeterminate future. Uncertainty exists as regards both the future and the past, but its nature is quite different in these two cases. The future is uncertain because it is not yet determined: it does not yet exist in a physical sense. Thus this uncertainty has an ontological character. The past however is fixed and unchanging, because it has already happened, and the time when it happened cannot be revisited; but our knowledge about it is incomplete, and can change with time. Thus this uncertainty is epistemological in nature. 


\section{Taking quantum physics seriously: emergence of certainty}

Quantum physics is, in crucial respects, very different from classical physics and we have to now take this into account. Quantum phenomena underlie classical phenomena, but often with completely counter-intuitive features. At the same time classical behaviour as we know it reliably emerges at everyday scales.

\subsection{Quantum features}

In the quantum world $[7,8,10,11,12,13,14,15,16,17]$ we must reconcile ourselves to that fact that:

- Only probabilities can be predicted, not specific outcomes;

- These probabilities are determined by a complex wave function or state vector, which evolves in a unitary fashion (a time-reversible linear process);

- The probabilities of measurement outcomes are given by the square of this state vector (this is a key non-linear feature of the theory);

- Superposition of states is allowed, indeed required, this process enabling interference and quantum tunneling to take place, and generically leading to quantum entanglement emerging;

- In an entangled state, ${ }^{2}$ state physical entities do not have separate individual existence; rather it is a collective state described by macroscopic variables;

- The potentialities of the quantum state are converted into the actualities of the classical state by a quantum measurement process that is not adequately described by current quantum theory; its existence and nature is a postulate over and above that of the current theory;

This process generically has a two-part nature:

- First is a process of environmental decoherence describable via unitary evolution, which effectively disentangles states and leads to the emergence of individual properties (decoherence converts entangled states to mixtures). The resulting state, however, while successfully predicting the probabilistic outcomes of statistical series of events,

\footnotetext{
${ }^{2}$ Two states are said to be "entangled" when they cannot be viewed as being independent of each other in any way, technically when their wavefunctions cannot be written as a simple product state.
} 
fails to predict the unique outcome of any specific event: it still represents a set of as yet unfulfilled potentialities;

- These potentialities are converted to a specific outcome by the second part of the measurement process: "collapse of the wave function" or "state vector reduction." This stage is where the central feature of quantum uncertainty manifests itself (this uncertainly never occurs in processes described only by the unitary evolution equations); thus this process can be thought of as actualizing potentialities;

- This second process is time-irreversible and causes information loss, and so is not describable by any unitary evolution;

- The two-part process is the generic case; however in specific cases, unique outcomes can occur where one or other of these measurement processes is not needed (i.e. classical outcomes can occur via unitary evolution). For example decoherence is not needed if the initial state is not entangled, and "wave function collapse" will not take place if the state vector is already in an eigenstate of the chosen operator;

- The measurement process resolves the dualities of quantum theory through choosing specific determinate outcomes, thus leading to classical states;

- It does so in a way that effectively can influence events that are apparently in the past, as is made manifest in delayed choice experiments (see $\S 4)$;

- The process of state preparation shares many of the properties of the process of measurement.

The upshot of this all is that weird quantum properties characterize matter before wave reduction take place; after it has taken place, the potential in its prior state is converted into definite outcomes. But the evolutionary equations of quantum theory are unitary, and hence entirely deterministic. Therefore the measurement process by which uncertainty is transmuted to certainty goes entirely undescribed by the theory itself. It is an extra feature outside quantum mechanics, but is essential for testing and interpreting the theory. Because the measurement process is crucial for the CBU picture, we devote the next section to outlining it. 


\subsection{Determinate to indeterminate}

The above considerations underscore the fact that we can't uniquely predict the future because of foundational quantum uncertainty relations (see e.g. $[11,12,16]$ ). We cannot predict precisely when a nucleus will decay or what the velocity of the resultant particles will be, nor can we predict precisely where a photon or electron in a double-slit experiment will end up on the screen. This unpredictability is not a result of a lack of information: it is the very nature of the underlying physics. More formally, consider the wave function or state vector $|\psi\rangle(x)$. The basic expansion postulate or quantum mechanics is that before a measurement is made, $|\psi\rangle$ can be written as a linear combination of eigenstates

$$
\left|\psi_{1}\right\rangle=\sum_{n} c_{n}\left|u_{n}(x)\right\rangle
$$

where $u_{n}$ is an eigenstate of some observable $\hat{A}$ (see e.g. [16]: 5-7). Immediately after a measurement is made at a time $t=t^{*}$, however, the wavefunction is found to be in one of the eigenstates:

$$
\left|\psi_{2}\right\rangle=c_{N} u_{N}(x)
$$

for some specific index $N$. The data for $t<t^{*}$ do not determine $N$; they merely determine a probability for the outcome $N$ through the fundamental equation

$$
p_{N}=c_{N}^{2}
$$

One can think of this as due to the probabilistic time-irreversible reduction of the wave function

$$
\begin{array}{ccc}
\left|\psi_{1}\right\rangle=\sum_{n} c_{n}\left|u_{n}(x)\right\rangle & \longrightarrow & \left|\psi_{2}\right\rangle=c_{N} u_{N}(x) \\
\text { Future : } & \text { Present : } & \text { Past : } \\
\text { Indeterminate } & \text { Transition } & \text { Determinate }
\end{array}
$$

([12]: 260-263). This is the event where the uncertainties of quantum theory become manifest (up to this time the evolution is determinate and time reversible). Invoking a many-worlds description (see e.g. [16]) will not help: in the actually experienced universe in which we make the measurement, $N$ is unpredictable (this proposal does not clarify in which branch of the wave function any specific observer will end up, so the experimental outcome (3.2) is unaltered by this hypothesis). 
A hidden variable theory may help, but here we will deal with standard quantum theory as determined by experiments. Thus the initial state (3.1) does not uniquely determine the final state (3.2); and this is not due to lack of data, it is due to the foundational nature of quantum interactions. You can predict the statistics of what is likely to happen but not the unique actual physical outcome, which unfolds in an unpredictable way as time progresses; you can only find out what this outcome is after it has happened. Furthermore, in general the time $t^{*}$ is also not predictable from the initial data: you don't know when the transition from (3.1) to (3.2) will take place, because you can't predict when a specific excited atom will emit a photon, or when a radioactive particle will decay.

We also can't retrodict to the past at the quantum level, because once the wave function has collapsed into an eigenstate it is impossible to determine from that eigenstate the configuration before the measurement. The fact that such events happen at the quantum level does not prevent them from having macro-level effects. Many systems can amplify quantum effects to macro levels, including photomultipliers (whose output can be used in computers or electronic control systems). Quantum fluctuations also change the genetic inheritance of animals, and so influence the course of evolutionary history on Earth. This is effectively what occurred when cosmic rays--whose emission processes are subject to quantum uncertainty — caused genetic damage in the distant past [6]. Furthermore, similar processes not only affect events in spacetime, but influence spacetime itself. For example, shortly after the big bang quantum fluctuations were amplified to astronomical scales by the universe's expansion, becoming seeds for galaxy formation (see [6]).

As regards the $\mathrm{CBU}$, the key feature is that not all transitions in quantum physics can be characterized by time-reversible Hamiltonian dynamics. This fundamental problem does not become evident if one is content to consider only statistical predictions for ensembles of identical microparticles: in that case environmental decoherence [18] results in diagonalization of the density matrix, giving the same statistical predictions for quantum and classical systems. But in the case of distinguishable particles where we want to determine the specific outcome for a single system, statistical predictions are inadequate and the collapse of the wave function to a specific eigenstate becomes germane; the collapse, as is well known, cannot be described by Hamiltonian dynamics. 


\section{Delayed choice experiments: Affecting the past}

Secondly, and more controversially, the collapse itself takes place in a way that, at least insofar as conventional language goes, appears to allow a degree of influence from the present to the past, reflecting the time-symmetric nature of the underlying physics. This is such an important feature of the Crystallizing Block Universe that we devote this section to the experimental evidence for such a claim and the next to theoretical attempts to come to grips with this phenomenon.

\subsection{Wheeler's delayed choice experiments}

The double slit experiment and its derivatives, particularly Wheeler's delayed-choice experiment [19], show that we are apparently able to influence the past to some degree, or at least that the conventional notion of "the past" is ill defined. The classic form of Wheeler's gedanken-experiment involves the usual double-slit configuration, in which a photon passes through the slits to fall on a detector or screen at some later time. As in the standard double-slit experiment, one can choose to measure the wave or particle property of the photon: measuring the photon's momentum (particle property) allows determination of the slit through which it passed but wipes out any information about its position (the interference pattern on the screen, a wave property). Measuring the interference pattern, on the other hand, destroys any information about the photon's momentum.

Wheeler's version of the experiment [19] added the striking feature that if the distance between the slits and the detector is sufficiently large, one can choose to measure the particle or wave property after the photon has passed the slits. How can we determine the photon's properties, after they should have already been decided? As counterintuitive as the situation may seem, over the past two decades laboratory confirmation of Wheeler's delayed-choice experiment has been achieved, in particular by the 2006 experiment of Jacques et al. [20], who attained levels of one photon in the apparatus at a given time, ruling out possible confusion in counting properties of different photons.

\subsection{Quantum erasers}

An equally, if not more bizarre, variant on the two-slit experiment proposed in 1982 by Scully and Drühl [21] has also been realized in the laboratory. The "quantum eraser" allows one to measure 
"simultaneously" both the slit through which a photon has passed and the interference pattern. To be sure, the position information (interference pattern) disappears when momentum ("which slit") information is obtained, but it reappears when the "which-slit" information is erased. The position information has not been destroyed by the momentum measurement, in contradiction to conclusions traditionally drawn from the Heisenberg uncertainty principle [22, 23, 24]. Moreover, the quantum eraser has also been performed in a delayed-choice version [25], in which the interference pattern can be erased or recovered even "after" the photon has been detected.

\subsection{Weak measurements}

A perhaps more well-known variety of experiment that throws doubt on the conventional interpretation of the past are the so-called "weak measurements" originally proposed by Aharonov, Albert and Vaidman [26]. Invariably, students are taught that any measurement of a quantum system collapses the wave function into an eigenstate. Weak measurements, as their name implies, disturb the system so little that no collapse in fact takes place. Furthermore, one is accustomed to think of the outcome of an experiment being determined by initial conditions. In quantum mechanics initial conditions are insufficient to determine the outcome of an experiment, due to the probabilistic nature of the theory; one needs both initial and final conditions to gain full knowledge. In devising a weak measurement, one prepares the system in a certain state (makes a "preselection"), later makes a final strong measurement ("postselection"). Both these initial and final boundary conditions determine the outcome of an intermediate ("weak") measurement. For example, if an ensemble of identical subsystems is preselected at an initial time $t_{0}$ such that $S_{z}=\uparrow$ and postselected at a later time $t_{2}$ such that $S_{x}=\uparrow$, then any measurement on an individual subsystem at an intermediate time $t_{1}$ must reveal $S_{z}=\uparrow$ (it was prepared to be so) as well as $S_{x}=\uparrow$ (otherwise the final measurement at $t_{2}$ would not get this result). For an arbitrary angle $\varphi$ in the x-z plane, however, $S_{\varphi}=S_{z} \sin \varphi+S_{x} \cos \varphi$. But $S_{z}=S_{x}=1 / 2$, and so a measurement at $\varphi=\pi / 4$ will yield $S_{\varphi}=\sqrt{2} / 2$, an apparently impossible result.

For textbook "strong" measurements, this is indeed impossible because $S_{z}$ and $S_{x}$ are noncommuting variables and hence a measurement on $S_{x}$ should "disturb" a measurement on the initial 
state, which is not an eigenstate of $S_{x}$. If, however, the initial state is indeed an ensemble consisting of many noninteracting replicas of the same subsystem (a product state), then a measurement on a single $S_{x}$ will be ignored by the vast majority of subsystems and the overall wavefunction will remain arbitrarily close to its initial state. The penalty one pays for registering both components of the spin is that the pointer of the device moves more than it should - the measurement looks like an error. Although one might balk at the notion of a final measurement influencing an intermediate measurement, the fact is, like the delayed-choice and eraser experiments, weak measurements have been experimentally observed (e.g. [27]) and, indeed, have recently provided a high degree of amplification in optical systems [28].

\section{$5 \quad$ Affecting the past: Quantum proposals}

The issue of delayed choice arises in regard to measurements that are made, and of course we don't yet have a fully satisfactory theory of quantum measurement: how the unitary evolution of the state vector changes to a single eigenstate when an observation is made. By contrast the unitary evolution between state measurements is well understood, as are the eigenvalues associated with particular outcomes, e.g. energy levels and scattering angles, and these are the main topic of most quantum theory textbooks. The issue then becomes that unitary time evolution is time symmetric: influences from the future should be as effective as influences from the past.

Some experiments, mentioned above, suggest that there are indeed real physical effects related to the time-reversed solutions: that in fact we can to some degree act on the past. Various theoretical efforts try to show how the measurement process could lead to such partially time symmetric outcomes. We here briefly mention two main classes of these theories; in a separate paper will analyze more closely their technical details and relationship to one another.

As a preliminary remark, we emphasize that any complete attempt to relate physical theory to the macro world must ultimately deal with individual systems and measurements as well as ensembles, because specific things happen to individual entities in the real world. Theories that deal with statistical predictions for ensembles of objects are very helpful in many contexts, but they simply 
do not succeed in giving unique predictions for specific objects; and a complete theory of the world should be able to do that. That is why an attempt to relate the theory to experiments, involving state vector reduction to a specific eigenstate, is essential.

\subsection{Transactional interpretations of quantum mechanics}

Many of the attempts to come to grips with the time-symmetric nature of quantum mechanics find their roots in the Wheeler-Feynman (WF) absorber theory of electromagnetic radiation [29, 30], which respects the time-symmetry inherent in Maxwell's equations. Developing earlier work by Tetrode and others, Wheeler and Feynman proposed a model where the field generated by an accelerated charge should consist of one-half the advanced plus one-half the retarded LiénardWiechert solutions of Maxwell equations. Thus, at $t=0$ the accelerated source charge emits a field that is $1 / 2($ advanced + retarded $)$. The retarded part reaches an absorber located at a distance $r$ after a time $r / c$. The absorber generates a field that is $1 / 2($ retarded - advanced $)$, which converges on the source at $t=0$. In other words, the advanced part of the wave generated by the absorber is $180^{\circ}$ out of phase with the advanced part generated by the source. Consequently, the advanced parts cancel and we are left with the usual retarded solution.

The WF theory is totally time-symmetric in that an advanced wave may be reinterpreted as a retarded wave by reversing the sign on $t$ and vice-versa. WF concluded that the irreversibility of the emission process must be "a phenomenon of statistical mechanics connected with the asymmetry of the initial conditions with respect to time." From their considerations, in particular of "preacceleration," Wheeler and Feynman conclude that "the past and future of all particles are tied together by a maze of interconnections" [29]. Nevertheless, Feynman later conceded [31] that the theory failed to give an adequate account of the self-energy of the electron, and it has been largely abandoned.

Although the WF theory was a theory of electromagnetism, Cramer [32, 33] has taken over its essentials for his "Transactional interpretation of quantum mechanics". He adopts $\psi^{*}(t)$, the solution to the conjugate equation, as the advanced wavefunction. Analogously to the WF picture, a quantum event is associated with a completed transaction. The retarded state vector extends an 
"offer wave," with amplitude $\psi(t)$. The absorber replies with an advanced "confirmation wave," $\psi^{*}(t)$, giving an amplitude back at the source (assuming no attenuation) of $P=\psi^{*} \psi$, which would be interpreted as the probability of the event. If there are many absorbers, the sum of all such offer-confirmation echoes is $\int \psi^{*} \psi d \tau$, the overlap integral.

The transactional interpretation can account for many quantum "paradoxes" in a fairly straightforward fashion. In the case of Wheeler's delayed-choice experiment, assume that if one wants to measure the wave properties of the photon, a photographic emulsion can be "instantly" put in place; to measure the momentum properties, the emulsion can be instantly lowered to reveal highly collimated CCD detectors, which record the "which-slit" information. For a position measurement in the transactional interpretation, a retarded offer wave travels through both slits to be absorbed at the emulsion. An advanced confirmation wave retraces the path through both slits to the source. Only "after" the confirmation wave reaches the source, the transaction is completed and the photon is said to have passed through both slits. In the complementary situation, the offer wave as before passes through both slits, but now the emulsion is lowered and the photon is recorded by one of the CCD detectors. The confirmation wave passes backwards through only one slit and the completed transaction is that characteristic of a single-slit event.

While the transactional interpretation is a bold development of the WF proposal, there are some difficulties with its interpretation; we deal with these in our technical companion paper.

\subsection{Two-time interpretations of quantum mechanics}

These interpretations are based in the idea, mentioned above, that one often deals with both preand post-selected ensembles, associated with state preparation and state measurement. For example, scattering experiments involve an initial beam, prepared to contain particles with a specific energy and momentum, and after the scattering event each detector measures the outgoing particles in another specific direction and energy range. Thus both preparation and measurement project into pre-determined eigenspaces of Hilbert space (note that the relevant operations do not necessary relate to a complete description of the state: they may leave some variables undetermined). The duality between pre- and post-selection reflects the time symmetry of the underlying theory. 
In 1964, Aharonov, Bergmann and Lebowitz ('ABL') [34] investigated the assertion that the "collapse of the wavefunction" introduces an arrow of time at a fundamental level in quantum mechanics. They found that, to the contrary, the arrow of time is introduced by the way statistical ensembles are constructed, and if time-symmetric boundary conditions are introduced, the resulting probability distributions are time symmetric as well. The ABL formula $[7,34]$ gives the probability that an intermediate measurement (between an initial and a final time) results in an eigenstate $a_{k}$ assuming that both initial and final states were specified:

$$
\operatorname{prob}\left(a_{k} \mid \psi_{i}, \psi_{f}\right)=\frac{\left|\left\langle\psi_{f} \mid a_{k}\right\rangle\right|^{2}\left|\left\langle a_{k} \mid \psi_{i}\right\rangle\right|^{2}}{\sum_{j}\left|\left\langle\psi_{f} \mid a_{j}\right\rangle\right|^{2}\left|\left\langle a_{j} \mid \psi_{i}\right\rangle\right|^{2}}
$$

where $\psi_{i}$ is the initial state and $\psi_{f}$ is the final state. Eq. (5.1) is the product of two conditional probabilities, the first giving the probability of getting $a_{k}$ given $\psi_{f}$ and the second giving the probability of getting $a_{k}$ given $\psi_{i}$. This formula shows that, to be sure, if final as well as initial conditions are specified, then the probability distribution is time symmetric. As already discussed in regard to weak measurements in $\S 4$, which were an outgrowth of the ABL formula, one specifies final boundary conditions in the same way as initial conditions: by selecting some members an ensemble on the basis of a specified property, eg. all electrons that are spin up at some final time. In the language of weak measurements one "preselects" initial states and "postselects" final states. In some sense this is a restatement of Gibbs' famous objection to the usual statistical mechanical argument for the increase of entropy. As Gibbs pointed out, given any low entropy state, entropy increases in both time directions; we, however, generally cut off the past; we predict rather than retrodict.

The ABL paper has been the subject of some controversy; we point out here only that Shimony [35] has vindicated the proposal by a rigorous derivation using Bayes theorem together with standard quantum mechanical predictions regarding ensembles that are only pre-selected.

In 2005 Aharonov and Gruss (AG, [36]; see also Section 18.3 of [7]) announced a two-time interpretation of quantum mechanics, developing from the work of ABL. Due to the first-order nature of the Schrödinger equation, one only has a single initial (or final) condition at one's disposal and so, if we impose both, we must have one state vector evolving forward in time and another 
evolving backward in time. They propose a History state vector $\left|\Psi_{H I S}(t)\right\rangle$ determined by the initial state $\left|\Psi_{H I S}\left(t_{0}\right)\right\rangle$ of the system and a Destiny state vector $\left\langle\Psi_{D E S}(t)\right|$ determined by its final state $\left\langle\Psi_{D E S}\left(t_{f}\right)\right|$. From the History and Destiny vectors they form the "two-state" density operator

$$
\rho(t)=\frac{\left|\Psi_{H I S}(t)\right\rangle\left\langle\Psi_{D E S}(t)\right|}{\left\langle\Psi_{D E S}(t)|| \Psi_{H I S}(t)\right\rangle}
$$

AG furthermore postulate that the system can be completely described by this operator, which evolves as

$$
\hat{\rho}\left(t_{2}\right)=U\left(t_{2}, t_{1}\right) \rho\left(t_{1}\right) U\left(t_{1}, t_{2}\right)
$$

where $U\left(t_{2}, t_{1}\right)$ is the standard unitary evolution operator $U=e^{-i H\left(t_{2}-t_{1}\right)}$.

The AG paper is in effect a restatement and development of a much earlier proposal by Davidon [37]; the relation between the two works will be discussed in our further paper. AG use the formalism to examine a spin-1/2 system from the two-time perspective and conclude that the description is entirely deterministic and local. They also show that two-time decoherence is completely timesymmetric, and go on to examine the implications for the two-time scenario when the final states are randomly distributed, leading to the possibility the weak measurements discussed in $\S 4$.

AG claim that the two-time formalism solves the quantum measurement problem: from their abstract, "the quantum superposition is, in effect, dynamically reduced to a single classical state via a 'two-time decoherence' process'." It is true that by imposing a final condition (say "up" for the apparatus in the destiny vector) one finds that the "two-state" operator (5.2), and hence the state of the system, must be measured to be "up" without any nonunitary evolution having taken place. However in order to recover the usual quantum probabilities during measurements on an ensemble, AG must assume a random distribution of final conditions. The assumed distribution of final conditions now determines the outcome of the measurements, and this is put in by hand. Thus, the AG proposal does not appear to adequately resolve the measurement problem, although it must be reemphasized that, regardless of interpretation, weak measurements have been observed.

More recently Davies [38] has used the two-time formalism to show that the textbook exponential 
decay law is in fact a restricted case of a more general quantum decay law that depends on both the time of post-selection and the final state, and has proposed that post-selection from the final state of the universe can be used to explain important features of the universe [39].

\subsection{Overall}

From the previous discussions, it is apparent that a number of serious proposals have been put forth that attempt to relate the time-symmetric unitary evolution of quantum theory to the possibility of the future influencing the past. Furthermore some of these theoretical explorations have led to new and notable experimental results. These attempts, however, do not express the passage of time as the EBU does. Many of them in fact assume that the future already exists (if the future did not already exist, it would of course not be able to influence the present or past). Our claim, by contrast, is that the future does not yet exist; at present the future is merely a set of possibilities. In the spirit of the EBU, any spacetime picture must be adopted to take this into account, as well as take into account the demands of quantum physics. Once one has this revised spacetime picture in mind, it should be possible to revisit all these theories and adapt them to a more realistic spacetime description. In the following sections we undertake to outline a spacetime description that unifies the backward-causality demonstrated in various quantum experiments with a realistic view of spacetime.

\section{The Crystallizing Block Universe}

The challenge is to modify the EBU picture of $\S 2$, to give a spacetime picture in which the paradoxical foundational features of quantum theory are taken seriously. In order to do so, we contemplate a crystallizing nature for the emergence of spacetime: not all features become fixed at the same time, and post-selection of previous events is possible. Potentiality changes to actuality at each quantum measurement process, but some potentialities may remain undecided even as others have transmuted to definiteness. Thus we consider that on a given world line "now" is the moment when those aspects of reality become fixed. On neighboring world lines the transition may occur at a later time and until the the transition takes place such aspects remain indefinite. The idea of 'post selection' then applies at the present time: we can now post-select states relating to interactions that have taken 
place in the past (as is made clear in the examples discussed by ABL).

The EBU evolves as discussed above, but does not settle all issues at once: some "events" that are left behind in the "past" remain uncertain, to become fixed only at a later time. An everyday analogy would be one of a crystallizing molten mixture: as the lattice of material becomes hardened and defines a fixed structure, some molten bits remain in the interstices to become fixed only later. The large scale structure crystallize out first and the inner details are filled in later; quantum uncertainty applies more to small objects than larger ones. The detailed past and future are separated by this crystallization surface ("CS"), rather than any macro approximation we make to it through a coarse-grained "surface of constant time."

The relation of quantum physics to the real universe is completely different to the past and the future of this surface: if we imagine a part of spacetime extending to the future of the CS, as a ghostly forerunner of the CBU spacetime domain, this region is subject to quantum uncertainty as expressed in the foundational probability equations (3.3) of quantum theory, and hence to all the associated feature of quantum duality. The part of spacetime to the past is not subject to any quantum uncertainty: all such uncertainty has been resolved, so the fundamental equation (3.3) is no longer applicable in that part of spacetime. It is replaced by the equation

$$
p_{n}=1(n=N), p_{n}=0(n \neq N)
$$

Thus quite different probabilistic equations hold in the past and the future of the CS (if we imagine spacetime extended to the future). This is why the ontological status of each domain is completely different.

The structure of spacetime is scale-dependent: when averaged on a large enough scale, this detailed micro-structure will be invisible, so on a coarse-grained view, we regain the classical EBU as described above. The description obtained thus depends on the level of detail one represents, with an averaged surface of concretization for the macro level (the macroscopic time "now") differing in detail from the microscopic surface CS where quantum uncertainty gives way to definiteness in a point by point way. 

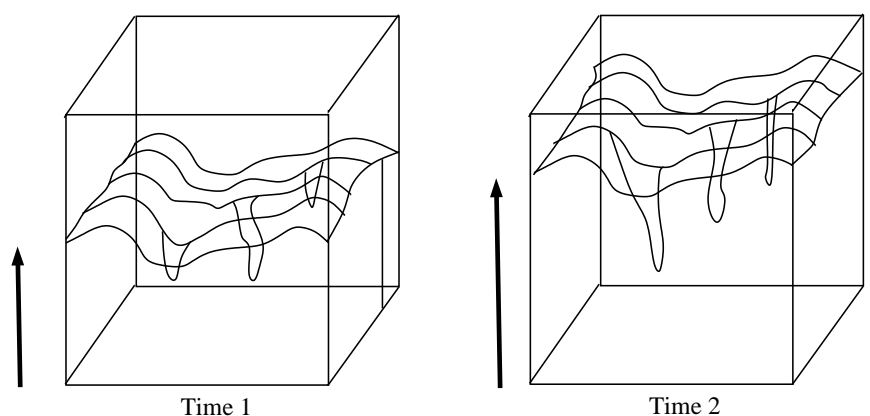

Figure 3: An evolving curved space-time picture that takes micro-phenomena seriously. Like the EBU (Figure 2), but here small pockets of potentiality remain unresolved till later times: delayed choice experiments can influence these 'past' events. The resolution of potential to definite outcomes takes place in a way like a crystallizing mixture, with pockets of residue remaining behind for a time after the main resolution front has passed.

\subsection{Experiments and Theory underlying the CBU}

The experiments underlying the CBU have already been described above, in $\S 4$. They seem to adequately validate the picture being put forward here, indeed they demand such a picture, whether we can provide an adequate theoretical explanation for these kinds of events or not.

Is there a viable theory underlying the CBU concept? A series of proposals have been described in $\S 5$ above. In brief: firstly, one can suggest that entanglement takes place in space and time, rather than just in space; secondly, decoherence can act apparently into the past as well as at a distance; and thirdly either a two-time or a transactional version of quantum theory promises to give an adequate theoretical underpinning for the experiments.

However there is one major factor to now be taken into account: those theories did not envisage a CBU context, and it is interesting to see how they must change when the CBU is introduced. Many of the proposals talk about final conditions for the universe; but we can only assign final conditions at the present time. Thus we envisage the possibility that either a transactional or a two-time formalism could underlie our proposal in a satisfactory way, but with " final conditions" imposed at the present time, instead of at the not-yet existing end of the universe. That suggestion is indeed largely satisfied already by the mathematical analyzes underlying the two sets of proposals, where there is in fact no specific mathematical feature characterizing the envisaged "final state" as necessarily existing "at the end of time."

Thus we suggest those analyzes can be adopted more or less as is, with the "final state" being 
taken as the present time $t_{0}$. Thus,

- It may possibly proceed as a variant of that described by Aharonov and Gruss [36], but with the later time in that formalism everywhere changed to the present day rather than the final state of the Universe, thus making their interpretation compatible both with the EBU idea and with realistic experimental testing.

- It may alternatively already be largely contained in the transactional viewpoint put by Cramer $[32,33]$, but now interpreted in the CBU context.

These proposals will be explored further in our follow-up paper.

\subsection{Measurement and the CBU}

The measurement interaction may perhaps be regarded as an interaction between scales. The measurement process itself is the crucial transition from indefinite to definite. On the face of it, this has the nature of top-down action from the macro level to the micro level [40], as is explicitly stated in the Copenhagen interpretation of quantum theory, and is implicit in most quantum physics writings (e.g. [11]), where a macroscopic detector is assumed to exist and give definite results. Decoherence [18] also has a top-down nature, expressed in the idea of environmental selection ('einselection') [41].

Thus the CBU may provide a sound context for expressing top-down causation from the macro to the micro level inherent in the measurement process, effective at the present time but with traces remaining undetermined until a later time. How this may be realised in detail needs to be pursued.

\subsection{The arrow of time}

As regards the arrow of time problem [42, 43], if the CBU view is correct, the Wheeler-Feynman prescription for introducing the arrow of time by integration over the far future [29], and associated views comparing the far future with the distant past [12, 44, 45], are invalid approaches to solving the arrow of time problem, for it is not possible to do integrations over future time domains if they do not yet exist. Indeed the use of half-advanced and half-retarded Feynman propagators in quantum field theory then becomes a calculational tool representing a local symmetry of the underlying physics that does not reflect the nature of emergent physical reality, in which that symmetry is broken. 
The arrow of time problem needs to be revisited in this CBU context. The key point is that the arrow of time arises simply because the future does not yet exist. One can be influenced at the present time from many causes lying in our past, as they have already taken place and their influence can thereafter be felt. One cannot be influenced by causes coming from the future, for they have not yet come into being. The history of the universe has brought the past into being, which is steadily extending to the future, and the future is just a set of unresolved potentialities at present. One cannot integrate over future events to determine their influence on the present not only because they do not yet exist, but because they are not even determined at present (as explained above in Section $\S 2)$.

The direction of the arrow of time is thus determined in a contingent way in the CBU context. Collapse of the quantum wave function is a prime candidate for a location of a physical solution to the arrow-of-time problem, and manifests itself as a form of time-asymmetric top-down action from the universe as a whole to local systems (cf. [12]). This takes place within the generic context of commonly occurring top-down action in the hierarchy of causality [40].

\section{Overall: A more realistic view}

The nature of the future is completely different from the nature of the past. When quantum effects are significant, the future manifests all the signs of quantum weirdness, including duality, uncertainty, and entanglement. With the passage of time, after the time-irreversible process of state-vector reduction has taken place, the past emerges, with the previous quantum uncertainty replaced by the classical certainty of definite particle identities and states. The present time is where this transition largely takes place. But the process does not take place uniformly or reversibly: evidence from delayed choice experiments shows that some isolated patches of quantum indeterminacy remain, and their transition from probability to certainty only takes place later. Thus, when quantum effects are significant, the Evolving Block Universe ("EBU") of classical physics cedes way to the Crystallizing

Block Universe ("CBU"). On large enough scales that quantum effects are not significant, the two models become indistinguishable. 
Crystallizing Universe...

Acknowledgements: We thank the NRF (South Africa) for support that made our collaboration on this project possible.

\section{References}

[1] D H Mellor, Real Time II. (Routledge, London: 1998).

[2] S Savitt, "Being and Becoming in Modern Physics", In The Stanford Encyclopedia of Philosophy (Spring 2002 Edition), Edward N. Zalta (ed.), see http://plato.stanford.edu/archives/spr2002/entries/spacetime-bebecome/.

[3] P C W Davies (2002), "That Mysterious Flow". Scientific American 287 (September), 40.

[4] H Price (1996) Time's Arrow and Archimedes' Point (New York: Oxford University Press).

[5] J B Barbour (1999) The End of Time: The Next Revolution in Phyiscs (Oxford: Oxford University Press).

[6] George F. R. Ellis (2006) "Physics in the Real Universe: Time and Spacetime". GRG 38:17971824 [http://arxiv.org/abs/gr-qc/0605049].

[7] Y Aharanov and D Rohrlich (2005): Quantum Paradoxes. Quantum Theory for the Perplexed (Weinheim: Wiley-VCH Verlag).

[8] G Greenstein and A G Zajonc (2006), The Quantum Challenge: Modern research on the Foundations of Quantum Mechanics (Sudbury, Mass: Jones and Bartlett Publishers).

[9] C D Broad (1923), Scientific Thought (New York: Harcourt, Brace and Co.). For Table of Contents and some chapters, see http://www.ditext.com/broad/st/st-con.html .

[10] F London, E Bauer (1983) "The theory of observation in quantum mechanics". In Quantum Theory and Measurement, J A Wheeler and W H Zurek (Eds), (Princeton: Princeton University Press), 217-259. 
[11] R Feynman (1985) QED: The Strange Theory of Light and Matter (Princeton: Princeton University Press).

[12] R Penrose (1989) The Emperor's New Mind (Oxford: Oxford University Press).

[13] M A Morrison (1990) Understanding Quantum Physics: a user's manual (Englewood Ciffs: Prentice Hall International).

[14] A J Leggett (1991) "Reflections on the Quantum Measurement Paradox". In Quantum Implications: Essays in Honour of David Bohm, Ed. B J Hiley and F D Peat (London: Routledge), $85-104$.

[15] A Rae (1994) Quantum Physics: Illusion or Reality? (Cambridge: Cambridge University Press).

[16] C J Isham (1997) Lectures on Quantum Theory: Mathematical and Structural Foundations (London: Imperial College Press).

[17] H.-P Breur, F Petruccione (2006) The Theory of open quantum systems (Oxford: Clarendon Press)

[18] C Kiefer (2002) "On the interpretation of quantum theory - from Copenhagen to the present day", arXiv:quant-ph/0210152v1.

[19] John Archibald Wheeler (1978), "The 'Past' and the 'Delayed-Choice Double-Slit Experiment'," pp 948, in A.R. Marlow, editor, Mathematical Foundations of Quantum Theory, Academic Press

[20] Vincent Jacques, E. Wu, Frdric Grosshans, Franois Treussart, Philippe Grangier, Alain Aspect, Jean-Franois Roch (2007). "Experimental realization of Wheeler's delayed-choice GedankenExperiment" Science 315, 5814. [arXiv:quant-ph/0610241v1]

[21] M.O. Scully and K. Drühl (1982), "Quantum eraser: A proposed photon correlation experiment concerning observation and 'delayed choice' in quantum mechanics" Phys. Rev. A 25, 2208 .

[22] Yakir Aharonov and M. Suhail Zubairy (2005) "Time and the Quantum: Erasing the Past and Impacting the Future" Science 307:875-879. 
[23] John G Cramer "The Quantum Eraser": http://www.npl.washington.edu/AV/altvw90.html,

[24] Ross Rhodes, commentary on "A Delayed Choice Quantum Eraser" : http://www.bottomlayer.com/bottom/kim-scully/kim-scully-web.htm.

[25] Yoon-Ho Kim, R. Yu, S.P. Kulik, Y.H. Shih, Marlan O. Scully [2000] "A Delayed Choice Quantum Eraser" Phys.Rev.Lett. 84:1-5 [arXiv:quant-ph/9903047v1]

[26] Yakir Aharonov, David Z. Albert, and Lev Vaidman (1986) "Measurement process in relativistic quantum theory" Phys. Rev. D 34, 1805 - 1813

[27] O. Hosten and P. Kwiat (2008) "Observation of the spin Hall effect of light via weak measurements" Science 319: 787.

[28] P. Ben Dixon, David J. Starling, Andrew N. Jordan, and John C. Howell (2009) "Ultrasensitive Beam Deflection Measurement via Interferometric Weak Value Amplification” Phys. Rev. Lett. 102, 173601.

[29] J. A. Wheeler and R. P. Feynman (1945), "Interaction with the Absorber as the Mechanism of Radiation". Rev. Mod. Phys. 17, 157-181.

[30] J. A. Wheeler and R. P. Feynman (1949), "Classical Electrodynamics in Terms of Direct Interparticle Action," Reviews of Modern Physics, 21, 425-433.

[31] R. P. Feynman (1949), "Space-time Approach to Quantum Electrodynamics", Phys. Rev. 76, 769-789.

[32] John G Cramer (1986) "The transactional interpretation of quantum mechanics" Rev of Mod Phys 58, 647-688.

[33] John G Cramer (1988) "An Overview of the Transactional Interpretation of Quantum Mechanics", Int Jour Theor Phys 27, 227

[34] Y Aharonov P G Bergmann J L Liebowitz (1964) "Time symmetry in the quantum process of measurement" Phys. Rev. 134, B1410 - B1416. 
[35] Abner Shimony (2005) "An Analysis of Ensembles that are Both Pre- and Post-Selected" Foundations of Physics 35 : 215-232

[36] Y Aharanov and E A Gruss (2005): "Two-time interpretation of quantum mechanics". arXiv:quant-ph/0507269.

[37] W C Davidon (1976) "Quantum Physics of single systems". Nuovo Cimento 36B: 34-39

[38] P C W Davies (2008), "Time dependent quantum weak values: decay law for post-selected states". arXiv:0807.1357

[39] Paul Davies (2006) The Goldilocks Enigma: Why Is the Universe Just Right for Life? (Allen Lane).

[40] G F R Ellis (2008) "On the nature of causation in complex systems" Trans Roy Soc South Africa 63: 69-84 .

[41] W Zurek (2004) "Quantum Darwinism and Envariance". In Science and ultimate reality: Quantum theory, Cosmology, and Complexity, J Barrow, P C W Davies and C Harper (Eds), (Cambridge University Press: Cambridge), 121-134.

[42] P C W Davies (1974), The physics of time asymmetry. (Surrey University Press, London).

[43] H D Zeh (1992), The Physical Basis of the Direction of Time. (Springer Verlag, Berlin).

[44] G F R Ellis and D W Sciama: "Global and non-global problems in cosmology". In General Relativity (A Synge Festschrift), ed. L. O'Raifeartaigh (Oxford University Press, 1972), 35-59.

[45] G F R Ellis (2002) "Cosmology and Local Physics". New Astronomy Reviews 46: 645-658 ( gr-qc/0102017). 